\title{
AUTOCONCEPTO E ÍNDICE DE MASA CORPORAL EN ESTUDIANTES UNIVERSITARIOS DEL 4to, 5to, 6to y 7 mo CICLO DE LA ESCUELA PROFESIONAL DE ENFERMERÍA DE LA UNIVERSIDAD ALAS PERUANAS, SANTIAGO DE SURCO, LIMA - PERU, 2016
}

\author{
SELF AND BODY MASS INDEX IN COLLEGE STUDENTS OF 4th, 5th, 6th and 7th \\ CYCLE OF PROFESSIONAL NURSING SCHOOL OF THE UNIVERSIDAD ALAS \\ PERUANAS, SANTIAGO DE SURCO, LIMA - PERU, 2016
}

\section{Rafael Garay Argandoña ${ }^{1, a}$, Jimmy Henry Baneo Pisco ${ }^{1, b}$}

\author{
${ }^{1}$ Escuela Profesional de Enfermería, Facultad de Medicina Humana y Ciencias de la Salud, \\ Universidad Alas Peruanas. Lima, Perú. \\ ${ }^{\mathrm{a}}$ Docente ; Magister. \\ ${ }^{\mathrm{b}}$ Estudiante
}

\section{Correspondencia}

Jimmy Henry Baneo Pisco

jbaneo8802_unap@hotmail.com

\section{RESUMEN}

La presente investigación, tuvo como; Objetivo: Establecer la relación del Autoconcepto con el Índice de Masa Corporal en los estudiantes universitarios del 4to, 5to, 6to y $7 \mathrm{mo}$ ciclo de la Escuela Profesional de Enfermería de la Universidad Alas Peruanas, Santiago de Surco, Lima - Perú, 2016. Materiales y métodos; se realizo un estudio de diseño descriptivo correlacional, de corte transversal y retrospectivo. La muestra estuvo conformada por 160 estudiantes de los distintos ciclos académicos. Los instrumentos utilizados fueron el cuestionario para determinar el autoconcepto y la ficha de de recolección de datos para IMC, se obtuvo una validez del $84 \%$ a través del método Delphi o juicio de expertos; con una confiabilidad de $75.4 \%$ para el cuestionario de autoconcepto, según el Alfa de Cronbach. Los datos se analizaron a través del programa estadístico 
SPSS versión 22. Para el análisis univariado, se empleó la estadística descriptiva para representar los datos expresados en frecuencias, porcentajes y tablas. En el análisis bivariado, se empleó la estadística inferencial, utilizando la prueba de Chi Cuadrado, para relacionar las variables en estudio con un $p<0,05$, como criterio de significancia. Resultados: De los 160 estudiantes (100\%); sobre el autoconcepto se observa que la mayor frecuencia de ellos 55.0\% (88 estudiantes) obtuvieron un Autoconcepto Positivo y solo $45.0 \%$ (72 estudiantes) un Autoconcepto Negativo. Sobre el IMC, se obtuvo que la mayor frecuencia de ellas 48.8\% (78 estudiantes) obtuvieron un diagnostico de Sobrepeso, otro grupo 40.8\% (64 estudiantes) se le diagnostico con un Peso normal y el $11.3 \% \%$ (18 estudiantes) con un diagnostico de Obesidad en relación al Índice de Masa Corporal. Se determino que existe relación entre el Autoconcepto y el Índice de Masa Corporal $(p=0,001 ; p<0,05)$.Conclusiones: Se comprueba relación estadísticamente significativa entre el Autoconcepto y el Índice de Masa Corporal; $p=0,001$ ( $p<0,05)$. El Autoconcepto positivo en estudiantes universitarios, es la categoría que mayor frecuencia tiene, con $55.0 \%$ de 88 estudiantes. En el Índice de Masa Corporal en estudiantes universitarios, se observa que la mayor frecuencia de ellas $48.8 \%$ (78 estudiantes) obtuvieron un diagnostico de Sobrepeso.

Palabras clave: Autoconcepto, sobrepeso, índice de masa corporal, obesidad.

\section{SUMMARY}

This research had as; Objective: To establish the relationship of Autoconcepto with Body Mass Index college students in 4th, 5th, 6th and 7th cycle of the Professional School of Nursing at the University Alas Peruanas, Santiago de Surco, Lima - Peru, 2016. Materials and methods; descriptive correlational study design, cross-cut was made retrospective. The sample consisted of 160 students from different academic cycles. The instruments used were the questionnaire I to determine the self-concept and tab data collection for BMI, a validity of $84 \%$ was obtained through the Delphi method or expert judgment; with a reliability of $75.4 \%$ for the self-concept questionnaire, according to the Cronbach's alpha. Data were analyzed through SPSS version 22. For univariate analysis, descriptive statistics were used to represent the data expressed in frequencies, percentages and tables. In bivariate analysis, inferential statistics was used, using Chi Square test to relate the study variables with $p<0.05$ as significance criterion. Results: Of the 160 students (100\%); on self-concept it shows that the most common of them $55.0 \%$ (88 students) had a positive self-concept and only $45.0 \%$ (72 students) A negative self-concept. On BMI, it was found that the most common of which $48.8 \%$ (78 students) had a diagnosis of overweight, another group $40.8 \%$ (64 students) was diagnosed with a normal weight and $11.3 \% \%$ (18 students) with a Obesity diagnosis in relation to body mass index. It was determined that there is a relationship between self-concept and body mass index $(p=$ 0.001; $p$ <0.05).Conclusions: Statistically significant relationship between self-concept and body mass index is checked; $p=0.001$ ( $p<0.05)$. The positive self-concept in college students is the category that most often is, with $55.0 \%$ of 88 students. In the Body Mass Index in college students, it is observed that the higher frequency of which $48.8 \%$ (78 students) had a diagnosis of overweight.

Keywords: Self-concept, overweight, body mass index, obesity. 


\section{INTRODUCCIÓN}

El presente estudio de investigación, se encuentra dentro del Área de Salud Comunitaria en Enfermería y en la línea de Alimentación y Nutrición.

En los últimos años, las investigaciones sobre el autoconcepto han proliferado en todos los países. El autoconcepto juega u papel decisivo y central en el desarrollo de la personalidad (1). El autoconcepto positivo o negativo incide en los resultados académicos, en la adaptación a situaciones nuevas, en el aprecio a su propio cuerpo, habilidades y comportamiento social (1). El autoconcepto comporta un proceso con trayectoria irregular, ya que sufre los periodos de estabilidad e inestabilidad características de cada una de las etapas del ciclo vital de los individuos (6).

Por ello el autoconcepto en términos valorativos, es el fin de autorreconocernos y autoaceptarnos, es claro también que el autoconcepto es dinámico y flexible, es decir es cambiante y se adapta a la etapa de vida en la se encuentra el individuo.

El índice de masa corporal es por tanto, una medida de proporcionalidad que permite establecerla adecuación del peso para la estatura. Nos indica la masa corporal distribuida en la superficie corporal del mismo (8). Un índice de masa corporal entre 20 y 25 está asociado con el menor riesgo de muerte temprana.

Por ello el índice de masa corporal es importante para determinar ciertos problemas de obesidad o de bajo peso en el individuo, esto nos lleva a que de esa forma podamos los profesionales de la salud brindar la dieta necesaria para el mejoramiento de la salud de la persona humana.

El interés que toma el curso de este trabajo de investigación, es el hecho de crear un interés sobre un estilo de vida saludable en lo que corresponde a la alimentación y nutrición, mas aun si los responsables de brindar dicha imagen somos los profesionales en la salud; solo así lograremos concientizar en la comunidad estudiantil dichos hábitos saludables, para que así podamos ir disminuyendo los índices de obesidad que se puedan presentar en algunos de nuestros estudiantes de la Escuela Profesional de Enfermería.

\section{MATERIALES Y MÉTODOS}

La presente investigación de acuerdo a su naturaleza tiene un enfoque cuantitativo de diseño descriptivo correlacional, ya que no se manipula la variable, la estudia tal cual se presenta, de corte transversal - observacional, porque busca relacionar las dos variables de investigación, se realiza dentro de un tiempo determinado; y de manera retrospectiva porque son datos ya dados.

La población de esta investigación estará constituida por los estudiantes universitarios del IV al VII ciclo de la Escuela Profesional de Enfermería siendo un total de 310 estudiantes matriculados.

\section{Criterios de Selección:}

- Todos los estudiantes matriculados en la escuela profesional de enfermería del IV 
al VII ciclo de la universidad Alas Peruanas.

- Los estudiantes que asistan regularmente a clases.

\section{Criterios de exclusión:}

- Los estudiantes retirados de la escuela profesional de enfermería.

- Los estudiantes que no desean participar en el estudio.

La muestra estará conformada por 160 estudiantes de los distintos ciclo en estudio del VI al VII ciclo, 40 participantes por ciclo.

El mecanismo utilizado para la recolección datos, consistió en aplicar una prueba con 18 preguntas, para evaluar el Autoconcepto, con opciones de respuesta tipo escala de lickert, distribuidas en tres dimensiones: Social, Emocional y Física. Para la evaluación del Índice de Masa Corporal, se utilizo una ficha de recolección de datos, para obtener información más precisa, cada participante debe ser tallado y pesado, para ello se utilizó un tallímetro y una balanza, se organizaron los datos según género y edad.

La información obtenida fue procesada, creándose una base de datos y utilizando para el análisis el paquete estadístico, en su versión SPSS 22.

Para el análisis descriptivo se trabajará tablas de frecuencia y gráficos de sectores y gráficos de barras en el programa Excel 2013.

Para el análisis bivariado se utilizara la prueba no paramétrica de chi cuadrado, para medir el grado de relación de las variables cualitativas.

\section{RESULTADOS}

De la Tabla № 1, Sobre el Autoconcepto en estudiantes universitarios del 4to, 5to, 6to y $7 \mathrm{mo}$ ciclo de la Escuela Profesional de Enfermería de la Universidad Alas Peruanas, Santiago de Surco, Lima Perú, 2016, de acuerdo a la prueba aplicada a $160(100,0 \%)$ estudiantes se observa que la mayor frecuencia de ellos $55.0 \%$ (88 estudiantes) obtuvieron un Autoconcepto Positivo y solo 45.5\% (72 estudiantes) un Autoconcepto Negativo.

De la Tabla № 2, Sobre el Índice de Masa Corporal en estudiantes universitarios del 4to, 5to, 6to y $7 \mathrm{mo}$ ciclo de la Escuela Profesional de Enfermería de la Universidad Alas Peruanas, Santiago de Surco, Lima - Perú, 2016, de acuerdo a la prueba aplicada a 160 (100,0\%) estudiantes, se observa que la mayor frecuencia de ellas 48.8\% (78 estudiantes) obtuvieron un diagnostico de Sobrepeso, otro grupo 40.8\% (64 estudiantes) se le diagnostico con un Peso normal y el $11.3 \% \%$ (18 estudiantes) con un diagnostico de Obesidad en relación al Índice de Masa Corporal.

De la Tabla № 3 Sobre el Autoconcepto e Índice de Masa Corporal en estudiantes universitarios del 4to, 5to, 6to y $7 \mathrm{mo}$ ciclo de la Escuela Profesional de Enfermería de la Universidad Alas Peruanas, Santiago de Surco, Lima Perú, 2016, se puede observar que de 64 
Tabla 1. Autoconcepto en estudiantes universitarios del 4to, 5to, 6to y $7 \mathrm{mo}$ ciclo de la Escuela Profesional de Enfermería de la Universidad Alas Peruanas, Santiago de Surco, Lima - Perú, 2016.

\begin{tabular}{lcc}
\hline \multicolumn{1}{c}{ AUTOCONCEPTO } & N & \% \\
\hline NEGATIVO & 72 & 45.0 \\
POSITIVO & 88 & 55.0 \\
Total & $\mathbf{1 6 0}$ & $\mathbf{1 0 0 . 0}$ \\
\hline
\end{tabular}

(40.0\%) estudiantes se les diagnostico con peso normal según el IMC, $28.8 \%$ (46 estudiantes) obtuvieron un autoconcepto positivo y $11.3 \%$ (18 estudiantes) obtuvieron un autoconcepto negativo. De los 78 (48.8\%) estudiantes se les diagnostico con sobrepeso según el IMC, $20.0 \%$ (32 estudiantes) obtuvieron un autoconcepto positivo y $28.8 \%$ (46 estudiantes) obtuvieron un autoconcepto negativo. De los 18 (11.3 $\%)$ estudiantes se les diagnostico con obesidad, $6.3 \% \quad(10 \quad$ estudiantes $)$ obtuvieron un autoconcepto positivo, 5.0 $\% \quad$ (8 estudiantes) obtuvieron un autoconcepto negativo.

De la Tabla № 4, La relación que existe entre el Autoconcepto y el Índice de Masa Corporal en estudiantes universitarios del 4to, 5to, 6to y $7 \mathrm{mo}$ ciclo de la Escuela Profesional de Enfermería de la Universidad Alas Peruanas, Santiago de Surco, Lima - Perú, 2016, se confirma con la prueba estadística no paramétrica de libre distribución Chicuadrada, cuyo valores fueron de Xc2 = $14,364, \mathrm{gl}=2 ; \mathrm{p}=0,001(\mathrm{p}<0,05)$, quedando demostrada la hipótesis planteada: "Existe relación entre el Autoconcepto y el Índice de Masa Corporal en estudiantes universitarios del 4to, 5to, 6to y $7 \mathrm{mo}$ ciclo de la Escuela

Profesional de Enfermería de la Universidad Alas Peruanas, Santiago de Surco, Lima - Perú, 2016.

Tabla 2. Índice de masa corporal en estudiantes universitarios del 4to, 5to, 6 to y $7 \mathrm{mo}$ ciclo de la Escuela Profesional de Enfermería de la Universidad Alas Peruanas, Santiago

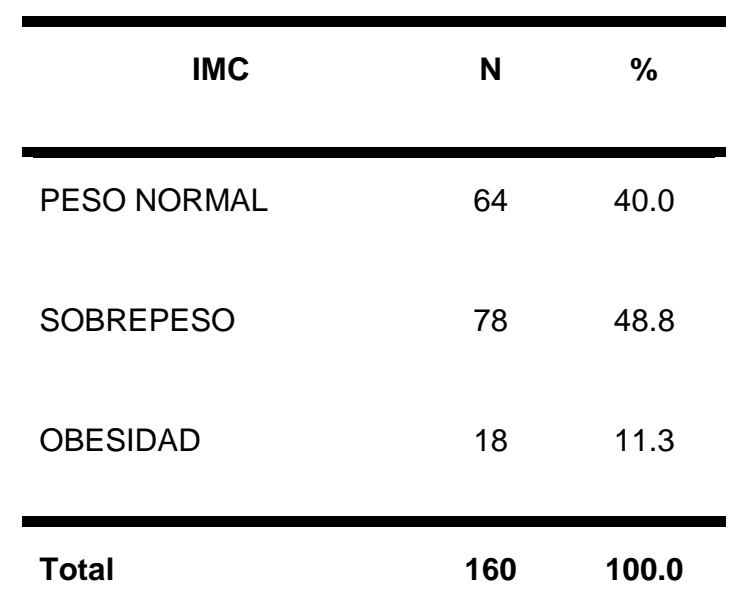

de Surco, Lima - Perú, 2016. 
Tabla 3. Autoconcepto e Índice de Masa Corporal en estudiantes universitarios del 4to, 5to, 6to y $7 \mathrm{mo}$ ciclo de la Escuela Profesional de Enfermería de la Universidad Alas Peruanas, Santiago de Surco, Lima Perú, 2016

Para encontrar la relación entre las dos variables de estudio se plantea lo siguiente:

- Formulación de hipótesis:

Ho: Hipótesis Nula:

No existe relación entre el Autoconcepto y el Índice de Masa Corporal en estudiantes universitarios del 4to, 5to, 6to y $7 \mathrm{mo}$ ciclo de la Escuela Profesional de Enfermería de la Universidad Alas Peruanas, Santiago de Surco, Lima - Perú, 2016.

Ha: Hipótesis Alterna:

$\mathrm{Si}$ existe relación entre el Autoconcepto y el Índice de Masa Corporal en estudiantes universitarios del 4to, 5to, 6to y $7 \mathrm{mo}$ ciclo de la Escuela Profesional de Enfermería de la Universidad Alas Peruanas, Santiago de Surco, Lima - Perú, 2016.

Nivel de Significancia: $(p=0,05)$; donde " $p$ " es llamado $p$ valor.

Toma de decisión:

$\mathrm{Si}$ el $(p<0,05)$, rechazamos la hipótesis nula; nos quedamos con la hipótesis alterna.
Tabla 4. Autoconcepto e Índice de Masa Corporal en estudiantes universitarios del 4to, 5to, 6to y $7 \mathrm{mo}$ ciclo de la Escuela Profesional de

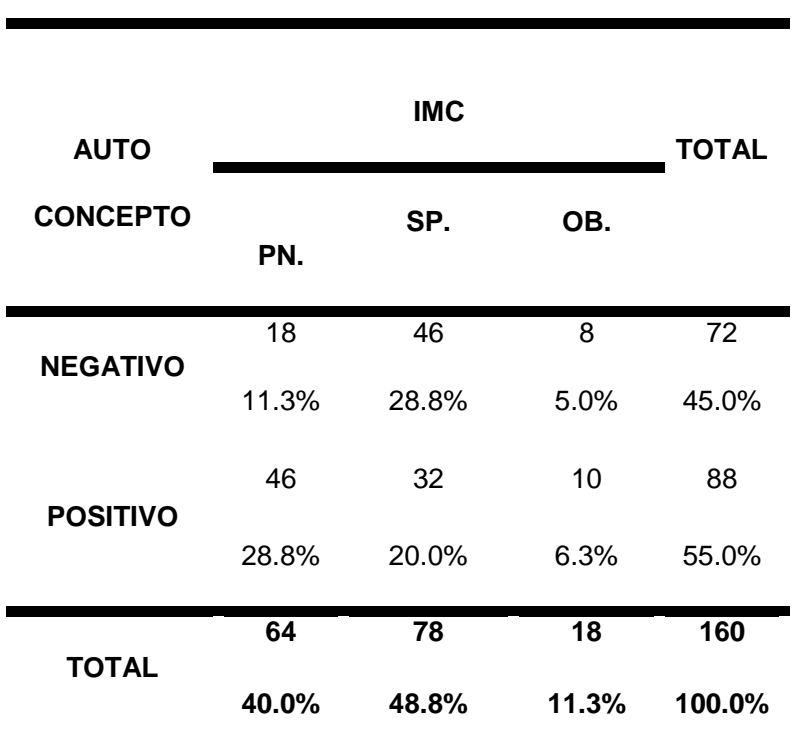

Enfermería de la Universidad Alas Peruanas, Santiago de Surco, Lima Perú, 2016.

\begin{tabular}{cccc}
\hline $\begin{array}{c}\text { Pruebas de } \\
\text { chi- } \\
\text { cuadrado }\end{array}$ & Valor & gl & $\begin{array}{c}\text { Sig. } \\
\text { asintótica } \\
\text { (2 caras) }\end{array}$ \\
\hline $\begin{array}{c}\text { Chi-cuadrado } \\
\text { de Pearson }\end{array}$ & $13,520^{\mathrm{a}}$ & 2 & .001 \\
$\begin{array}{c}\text { Razón de } \\
\text { verosimilitud }\end{array}$ & 13.821 & 2 & .001 \\
$\begin{array}{c}\text { Asociación } \\
\text { lineal por } \\
\text { lineal }\end{array}$ & 6.684 & 1 & .010 \\
\hline $\begin{array}{c}\mathrm{N} \text { de casos } \\
\text { válidos }\end{array}$ & 160 & & \\
\hline
\end{tabular}

\section{DISCUSION}


De acuerdo a los objetivos planteados en la presente investigación, se estableció la relación de el Autoconcepto y el Índice de Masa Corporal en estudiantes universitarios del 4to, 5to, 6to y $7 \mathrm{mo}$ ciclo de la Escuela Profesional de Enfermería de la Universidad Alas Peruanas, Santiago de Surco, Lima - Perú, 2016

Al analizar el Autoconcepto y el Índice de Masa Corporal en estudiantes universitarios del 4 to, 5to, 6to y $7 \mathrm{mo}$ ciclo de la Escuela Profesional de Enfermería de la Universidad Alas Peruanas, se confirma con la prueba estadística no paramétrica de libre distribución Chicuadrada, cuyo valores fueron de Xc2 = $14,364, g l=2 ; p=0,001 \quad(p<0,05)$, quedando demostrada la hipótesis planteada: "Existe relación entre el Autoconcepto y el Índice de Masa Corporal", estos resultados son similares a los encontrados por GARRIDO CEDILLO, C.M (2014), en su estudio "Relación del autoconcepto y el índice de masa corporal (IMC), en una muestra de estudiantes universitarios por género masculino y femenino". Universidad Pública del Estado de México. Los resultados de correlación mostraron, que al hacer la relación por genero, encontramos que las féminas reportaron una relación significativamente mayor entre el autoconcepto físico y el IMC sin alteraciones en el autoconcepto general. En cuanto al género masculino no se encontró relación entre el IMC y el autoconcepto físico.

Al analizar el Autoconcepto y el Índice de Masa Corporal en estudiantes universitarios del 4 to, 5to, 6to y $7 \mathrm{mo}$ ciclo de la Escuela Profesional de Enfermería de la Universidad Alas Peruanas, se observa que la mayor frecuencia de ellas
$48.8 \% \quad$ (78 estudiantes) se les diagnostico Sobrepeso, otro grupo $40.8 \%$ (64 estudiantes) se le diagnostico Peso normal y el $11.3 \% \%$ (18 estudiantes) con un diagnostico de Obesidad en relación al Índice de Masa Corporal, además se confirma con la prueba estadística no paramétrica de libre distribución Chicuadrada, cuyo valores fueron de Xc2 = $14,364, \mathrm{gl}=2 ; \mathrm{p}=0,001 \quad(\mathrm{p}<0,05)$, quedando demostrada la hipótesis planteada: "Existe relación entre el Autoconcepto y el Índice de Masa Corporal; estos resultados son similares a los encontrados por, Alcaraz López, G; Lora Gómez, E; Berrio Estrada, M.E (2007), en su estudio "Índice de masa corporal y percepción de la Imagen corporal en estudiantes de enfermería. Facultad de Enfermería, Universidad de Antioquia, Medellín - Colombia". El análisis de IMC muestra un promedio de 22,7, mínimo 17,0 y un máximo de 31,7: $26,6 \%$ de las estudiantes presentan pesos por encima o por debajo del peso ideal. Respecto a la percepción de la imagen corporal: $4,5 \%$ de las estudiantes se perciben bajas de peso siete de cada $10(68,4 \%)$ se percibieron en sobrepeso y obesidad. El $68,4 \%$ de las estudiantes sobreestiman su imagen corporal $(p=$ $0,05)$ y $4,5 \%$ la subestiman. Hubo concordancia entre el IMC real y la percepción de la imagen corporal solo en $38,2 \%$.

Al analizar el Autoconcepto y el Índice de Masa Corporal en estudiantes universitarios del 4to, 5to, 6to y $7 \mathrm{mo}$ ciclo de la Escuela Profesional de Enfermería de la Universidad Alas Peruanas; se observa que la mayor frecuencia de ellas $48.8 \%$ (78 estudiantes) obtuvieron un diagnostico de Sobrepeso, otro grupo $40.8 \%$ (64 estudiantes) se le diagnostico 
con un Peso normal y el $11.3 \% \%$ (18 estudiantes) con un diagnostico de Obesidad en relación al Índice de Masa Corporal; estyos resultados son similares a los encontrados por, Miraval Rojas, E.J; Greiner Ponce, A; Perea Villafranca, E; Suarez, Z; Calderón Iparraguirre, M; Hernández, L, Escorza Hoyle, A.M; Ojeda, L. (2013). Relación entre el índice de masa corporal y la percepción de imagen corporal en universitarias. Lima Perú. Entre los resultados obtenidos se observo que según el índice de masa corporal, se encontró una prevalencia de bajo peso de $3 \%$, normal $71 \%$, sobrepeso $26 \%$.

\section{CONCLUSIONES}

Se comprueba relación estadísticamente significativa entre el Autoconcepto y el Índice de Masa Corporal en estudiantes universitarios del 4to, 5to, 6to y $7 \mathrm{mo}$ ciclo de la Escuela Profesional de Enfermería de la Universidad Alas Peruanas, Santiago de Surco, Lima - Perú, 2016, se confirma con la prueba estadística no paramétrica de libre distribución Chicuadrada, cuyo valor hallado fue; $p=$ $0,001(p<0,05)$, quedando demostrada la hipótesis planteada.

El Autoconcepto en estudiantes universitarios, según las categorías analizadas es con mayor frecuencia Positivo; $55.0 \%$ de 88 estudiantes obtuvieron ese resultado Positivo, y solo $45.5 \%$ de 72 estudiantes un Autoconcepto Negativo.

Sobre el Índice de Masa Corporal en estudiantes universitarios, se observa que la mayor frecuencia de ellas $48.8 \%$ (78 estudiantes) obtuvieron un diagnostico de Sobrepeso, otro grupo $40.8 \%$ (64 estudiantes) se le diagnostico con un Peso normal y el $11.3 \% \%$ (18 estudiantes) con un diagnostico de Obesidad en relación al Índice de Masa Corporal.

\section{FUENTE DE FINANCIAMIENTO}

Autofinanciado

\section{CONFLICTOS DE INTERÉS}

E I autor declara no tener conflictos de interés en la publicación de este artículo.

\section{REFERENCIAS BIBLIOGRÁFICAS}

1. GARRIDO CEDILLO, C.M. Relación del autoconcepto y el índice de masa corporal (IMC), en una muestra de estudiantes universitarios por género masculino y femenino. Int. med. cienc. act. fis. deporte. 2016 Junio.

2. Alcaraz López, G; Lora Gómez, E; Berrio Estrada, ME . Índice de masa corporal y percepción de la Imagen corporal en estudiantes de enfermería. Index de Enfermeria. 2011 Junio; 20(1 - 2).

3. Miraval Rojas, E.J; Greiner Ponce, A; Perea Villafranca, E; Suarez, Z; Calderón Iparraguirre, M; Hernández, L, Escorza Hoyle, A.M; Ojeda, L. Relación entre el índice de masa corporal y la percepción de imagen corporal en universitarias. Concensus. 2014. 19 (2).

4. Huerta Benel, R; Campos Campos, S; Cruzado, L (2012). Insatisfacción corporal en estudiantes de medicina de la 
Universidad Peruana Cayetano Heredia medida con el Body Shape Questionnaire. Neuropsiquiatr 75. 2012 (3).

5. Promoción de la salud. Nola Pender. Disponible en internet URL:

htpps//sites.google.com/site/todop araenfermeria/teorías - y modelos d enfermería. Consultado el 12 de octubre del 2015.

6. Linares Manrique, M; Linares Gírela, D; Matos Medina, O; Cruz Quintana, F. Relación entre el Auto concepto Físico, Ansiedad e IMC en estudiantes universitarios mexicanos. Int. med. cienc. act. fis. deporte. 2016 Junio.

7. Manual AF5. Disponible en internet URL: htpps//web.teaediciones.com/af5-autoconcepto-forma--.-5.aspx

8. Carrasco; R; Fernando $\mathbf{Y}$ Baldeon. Manual E. Prevalencia de sobrepeso y obesidad en estudiantes adolescentes ecuatorianos del área urbana. ALAN (ONLINE). 2011, vol 58, n.2.

9. Garay Sánchez, G. Sobre peso y obesidad en el universitario: Implicaciones en la consejería. Rev Griot, Diciembre 2011, vol 4, $\mathrm{n}^{\circ} 1$.

10. Ma. Refugio Ríos Saldaña (2015). Estilo de vida y obesidad en estudiantes universitarios: una mirada con perspectiva de género. Facultad de Estudios Superiores Iztacala UNAM. México.
11. Luis Gabriel Rangel Caballero, Lyda Zoraya Rojas Sánchez y Edna Magaly Gamboa Delgado. (2015). Sobrepeso y obesidad en estudiantes universitarios colombianos y su asociación con
12. Luis Gabriel Rangel Caballero, Lyda Zoraya Rojas Sánchez y Edna Magaly Gamboa Delgado. (2015). Sobrepeso y obesidad en estudiantes universitarios colombianos y su asociación con la actividad física. Universidad Santo Tomás. Bucaramanga. Fundación Cardiovascular de Colombia. Colombia.

13. M. M. Rizo-Baeza, N. G. González - Brauery E. Cortés (2014). Calidad de la dieta y estilos de vida en estudiantes de Ciencias de la Salud. Departamento de Enfermería. Universidad de Alicante. Departamento de Farmacología, 


\section{Pediatría y Q. Orgánica. \\ Universidad Miguel Hernández. \\ Alicante. España.}

Citar como: GARAY ARGANDOÑA, R. BANEO PISCO, JH.

Autoconcepto e Índice de masa corporal en estudiantes universitarios del 4to, 5to, 6to y $7 \mathrm{mo}$ ciclo de la Escuela Profesional de Enfermería de la Universidad Alas Peruanas, Santiago de Surco, Lima - Perú, 2016. 